\title{
Baroclinic instability of a symmetric, rotating, stratified flow: a study of the nonlinear stabilisation mechanisms in the presence of viscosity
}

\author{
R. Mantovani ${ }^{1, *}$ and A. Speranza ${ }^{1}$ \\ ${ }^{1}$ Mathematics Department, University of Camerino, Via Madonna delle Carceri, 62032 Camerino (MC), Italy \\ * presently: ESRIN, European Space Agency, Via Galileo Galilei, 00044 Frascati, Italy
}

Received: 25 January 2002 - Revised: 8 May 2002 - Accepted: 17 June 2002

\begin{abstract}
This paper presents the analysis of symmetric circulations of a rotating baroclinic flow, forced by a steady thermal wind and dissipated by Laplacian friction. The analysis is performed with numerical time-integration. Symmetric flows, vertically bound by horizontal walls and subject to either periodic or vertical wall lateral boundary conditions, are investigated in the region of parameter-space where unstable small amplitude modes evolve into stable stationary nonlinear solutions. The distribution of solutions in parameter-space is analysed up to the threshold of chaotic behaviour and the physical nature of the nonlinear interaction operating on the finite amplitude unstable modes is investigated. In particular, analysis of time-dependent energyconversions allows understanding of the physical mechanisms operating from the initial phase of linear instability to the finite amplitude stable state. Vertical shear of the basic flow is shown to play a direct role in injecting energy into symmetric flow since the stage of linear growth. Dissipation proves essential not only in limiting the energy of linearly unstable modes, but also in selecting their dominant spacescales in the finite amplitude stage.
\end{abstract}

\section{Introduction}

Although never directly observed, in nature or in laboratory, symmetric baroclinic instability has been considered for decades a natural physical process of potential relevance in geophysical and astrophysical fluid dynamics. For what concerns its role in Meteorology, the difficulties that have to be faced in the application of the available theoretical concepts in the context of operative analysis-forecasting activity have been extensively illustrated in literature (see, for example, the recent review by Schultz and Schumacher, 1999). As a matter of fact, our interest is particularly in the potential role of symmetric baroclinic instability in some weather phe-

Correspondence to: A. Speranza

(antonio.speranza@unicam.it) nomena typical of the Mediterranean area. It is known that (usually under cyclogenetic conditions) "banners" of negative potential vorticity can form downstream of the Alps (see, for example, Aebischer and Schär, 1998) and intense precipitations can take place even under conditions of overall stability with respect to ordinary convection (see, for example, Tudur and Ramis, 1997) within (usually sub-frontal) "precipitation bands". This induces to assume that sub-synoptic scale processes of vertical uplift may be operating in the form of "symmetric" (almost two-dimensional) flow: symmetric baroclinic conversion is, consequently, a natural candidate. However, despite the potential practical importance and the numerous published studies, a solid theoretical background concerning quasi-two-dimensional baroclinic conversion is still lacking. Particularly needed is a deeper understanding of the physical nature of the finite amplitude stabilisation mechanism, not only for its obvious impact on the "parameterisation" of symmetric instability, but also for its importance in interpreting observations and designing new observation devices (in particular remote sensing from space).

In view of the above mentioned problems, we analyse in this paper an idealised fluid system often discussed in literature (see, in particular, Gu et al., 1998; hereinafter GXW), nonlinear viscous symmetric flow of a rotating Boussinesq fluid. The symmetric flow is "forced" by the baroclinic instability of the basic flow (a uniform thermal wind in the direction of symmetry kept constant in time) and dissipated by diffusive (Laplacian) friction. The time-evolution is explored, both in the linear and nonlinear regimes of flow, by means of spectral and finite-difference numerical models. Several physical properties of the flow evolution (space-scales, energy, potential vorticity, etc.), as well as the distribution of solution types (stable linear, stable nonlinear, chaotic) in the parameter space are analysed and discussed. The main objective is to capture the essential physical nature of the stabilisation mechanism.

The paper is organised as follows: in Sect. 2 the basic equations are proposed together with a description of the physical system they model; in Sect. 3 and 4 numerical 
experiments on time-evolution of the flow under, respectively, periodic and "box" lateral boundary conditions are presented; in Sect. 5 the physical nature of the mechanisms operating in the numerical time-evolution is further investigated by means of energy analysis; in Sect. 6 we draw our conclusions.

\section{Equations and physical model}

\subsection{Basic state and perturbation equations}

The model adopted in the present work is that proposed by GXW where the fluid flow variables are represented in terms of a basic state and a superimposed perturbation. The basic state is a flow in the $y$-direction (the symmetry direction), with a constant vertical shear, uniform in the other two directions and in thermal wind equilibrium:

$V=(z-H / 2) V_{z}$,

where $V_{z}$ is the constant vertical shear and $H$ is the height of the domain. Because of the thermal wind balance the following relationship holds between vertical wind shear and horizontal temperature gradient:

$f V_{z}=\gamma \partial \Theta / \partial x=S^{2}$,

where $f$ is the (constant) Coriolis parameter; $\gamma=g / \Theta_{0}$, with $g$ gravity acceleration and $\Theta_{0}$ a reference potential temperature; $\Theta$ is the potential temperature of the basic state defined as:

$\Theta=\Theta_{0}+\left(x S^{2}+z N^{2}\right) \gamma^{-1}$,

where $N^{2}=\gamma \partial \Theta / \partial z$ is the constant vertical stratification of the basic state.

The equations governing the time evolution of the perturbation are the Boussinesq equations:

$\frac{d u}{d t}-f v=-\frac{1}{\rho_{0}} \partial_{x} p+\eta \nabla^{2} u$,

$\frac{d v}{d t}+f u+w V_{z}=-\frac{1}{\rho_{0}} \partial_{y} p+\eta \nabla^{2} v$,

$\frac{d w}{d t}=-\frac{1}{\rho_{0}} \partial_{z} p+b+\eta \nabla^{2} w$,

$\frac{d b}{d t}+u S^{2}+w N^{2}=\eta \nabla^{2} b$,

$\partial_{x} u+\partial_{y} v+\partial_{z} w=0$

where $u, v$, and $w$ are the three components of the wind vector, $p$ is the pressure, $\rho_{0}$ is a constant reference density, $b=$ $\gamma \vartheta$ is the buoyancy associated with the perturbation potential temperature $\vartheta, \eta$ is the turbulent diffusion coefficient, $\nabla^{2}=$ $\partial_{x x}^{2}+\partial_{y y}^{2}+\partial_{z z}^{2}$, and $d / d t=\partial / \partial t+u \partial_{x}+(v+V) \partial_{y}+w \partial_{z}$.

The applied boundary conditions are non-slip, conductive in the vertical direction:

$u=v=w=b=0$ at $z=0, H ;$ and periodic or non-slip, conductive in the horizontal direction.

The differential problem is made non-dimensional with the following parameters: $H$ is the vertical scale, $N H / f$ the horizontal scale, $f^{-1}$ the time-scale, $H V_{z}$ the horizontal velocity scale, $H V_{z} f / N$ the vertical velocity scale, $\rho_{0} N H^{2} V_{z}$ the pressure scale and $N H V_{z}$ the buoyancy scale. The following "numbers" appear in the non-dimensional formulation of the Boussinesq equations:

- Richardson number: $\mathrm{Ri}=N^{2} / V_{z}^{2}$,

- Rossby number: Ro $=V_{z} / f$,

- Ekman number: $\mathrm{Ek}=\eta /\left(f H^{2}\right)$.

Choosing the parameter values (standard in literature on the subject): $f=10^{-4} \mathrm{~s}^{-1}, \eta=100 \mathrm{~m}^{2} \mathrm{~s}^{-1}, V_{z}=10 \mathrm{~km}$ and $\mathrm{km}$, the Rossby number and the Ekman number respectively assume the values $\mathrm{Ro}=10$ and $\mathrm{Ek}=10^{-2}$, while the Richardson number is maintained variable. Only in a particular case presented in Sect. 5, different values of the Ekman number are adopted.

\subsection{Two-dimensional equations}

Introducing the symmetry condition $\left(\partial_{y}=0\right)$ and defining a streamfunction for two-dimensional flow in the $x-z$ plane by means of the relationships $u=\partial_{z} \Psi$ and $w=-\partial_{x} \Psi$, Eqs. (4a) and (4c) can be combined to eliminate the pressure from the problem. Three prognostic equations for the vorticity, $\Delta \Psi=\left(\partial_{x x}^{2}+\partial_{z z}^{2}\right) \Psi$, the velocity component in the $y$-direction, $v$, and the buoyancy, $b$, are then obtained:

$\partial_{t} \Delta \Psi+J(\Psi, \Delta \Psi)+\partial_{x} b-f \partial_{z} v=\eta \Delta^{2} \Psi$,

$\partial_{t} v+J(\Psi, v)+f \partial_{z} \Psi-V_{z} \partial_{x} \Psi=\eta \Delta v$,

$\partial_{t} b+J(\Psi, b)+S^{2} \partial_{z} \Psi-N^{2} \partial_{x} \Psi=\eta \Delta b$,

where $J(\Psi, \cdot)=\partial_{z} \Psi \partial_{x}-\partial_{x} \Psi \partial_{z}$ and $\Delta^{2}=\left(\partial_{x x}^{2}+\partial_{z z}^{2}\right)^{2}$. In this vorticity-streamfunction formulation the vertical boundary conditions are:

$\Psi-\partial_{z} \Psi=v=b=0$ at $z=0, H$,

and the horizontal boundary conditions are: periodic in

$x$,

or

$\Psi=\partial_{x} \Psi=v=b=0$ at $x=0, L$

$L$ being the extension of the horizontal domain in the $x$ direction. 


\subsection{The physical model}

As already mentioned, the differential problem proposed above is standard in literature on the subject. However it is useful, in view of successive discussion, to describe here explicitly the physical nature of the system modelled by equations $(6 a-c)$ and boundary conditions (7a), (7b) or (7a), (7c).

The system is forced by the instability of the steady, uniform thermal wind and dissipated by Laplacian friction. The basic flow is clearly inconsistent with the boundary conditions (see, for example, Miller, 1984 for a discussion of this aspect of the problem): it is used as an artifice for forcing the system by means of the energy conversion from the basic state into the perturbation field associated with linear instability. With the prescribed boundary conditions the symmetric perturbations cannot change the overall gradients of potential temperature and angular momentum which, consequently, remain the ones of the basic state.

One immediate consequence of this approach is that, in case of instability, linear baroclinic adjustment (Stone, 1978) of the basic state, i.e. evolution of the system towards the threshold of linear baroclinic instability of the basic state, is not allowed since the "global" stress parameter of the system, the Richardson number, cannot be changed by internal perturbations. Only the nonlinear deformation of the flow (taking place in a subspace of phase-space with no projection onto the overall gradients of the basic state) can stabilise the system. Since the instability of the basic flow cannot be neutralised, only frictional dissipation can close the energy balance and compensate energy injection by unstable conversion.

\section{The periodic configuration}

\subsection{Spectral representation}

When horizontally periodic boundary conditions are adopted, the following spectral representation is selected:

$\Psi(x, z, t)=\sum_{m=0, n=1}^{\infty, \infty} \Psi_{m, n}(t) f_{m}(x) g_{n}(z)$,

$v(x, z, t)=\sum_{m=0, n=1}^{\infty, \infty} v_{m, n}(t) f_{m}(x) q_{n}(z)$,

$b(x, z, t)=\sum_{m=0, n=1}^{\infty, \infty} b_{m, n}(t) f_{m}(x) q_{n}(z)$,

where:

$f_{m}(x)= \begin{cases}\cos \frac{m}{2} k x & \text { for even } m, \\ \sin \frac{m+1}{2} k x & \text { for odd } m,\end{cases}$

$q_{n}(z)=\sin (n \pi z / H)$,

$g_{n}(z)=\left\{\begin{array}{l}\frac{\sinh \left(\mu_{n / 2} \bar{z}\right)}{\sinh \left(\mu_{n / 2} / 2\right)}-\frac{\sin \left(\mu_{n / 2} \bar{z}\right)}{\sin \left(\mu_{n / 2} / 2\right)} \text { for even } n, \\ \frac{\cosh \left(\lambda_{n / 2} \bar{z}\right)}{\cosh \left(\lambda_{n / 2} / 2\right)}-\frac{\cos \left(\lambda_{n / 2} \bar{z}\right)}{\cos \left(\lambda_{n / 2} / 2\right)} \text { for odd } n .\end{array}\right.$ $\bar{z}=z / H-0.5$ and the coefficients $\mu_{n / 2}$ and $\lambda_{(n+1) / 2}$ are fixed by the following equations (derived from the streamfunction boundary conditions):

$\operatorname{coth} \frac{1}{2} \mu_{n / 2}-\cot \frac{1}{2} \mu_{n / 2}=0$ for even $n$,

$\tanh \frac{1}{2} \lambda_{(n+1) / 2}+\tan \frac{1}{2} \lambda_{(n+1) / 2}=0$ for odd $n$.

It is important to note that the functions (9c) chosen for the spectral representation of the streamfunction are not the natural eigenfunctions of the differential problem obtained by linearisation of $(6 a-c),(7 a),(7 b)$; they are simply a complete set of eigenfunctions of the lowest order linear differential (in the vertical coordinate) problem satisfying the chosen boundary conditions (Emanuel, 1979). As a consequence, we have, even in the near-critical regime, no mathematical theory supporting us in estimating a priori numerical convergence and other properties of the spectral numerical representation. Specifically, convergence of the spectral representation must be estimated empirically, on the basis of changes in the numerical results from one order of expansion to the other.

To set up the spectral model, the series $(8 \mathrm{a}-\mathrm{c})$ is substituted into the system $(6 \mathrm{a}-\mathrm{c})$ and, projecting (6a) onto $f_{i} g_{j}$ and (6b), (6c) onto $f_{i} q_{j}$ and truncating, a closed system of ordinary differential equations for the spectral coefficients $\Psi_{i, j}(t), v_{i, j}(t)$ and $b_{i, j}(t)$ is obtained: this system is integrated by means of a fourth order Runge-Kutta scheme.

\subsection{Linear regime}

Linear perturbation equations are obtained by eliminating the Jacobian $J(\Psi, \cdot)$ in $(6 \mathrm{a}-\mathrm{c})$. For the truncated spectral expansion, eight functions of $z$ are used; as mentioned above, the number is fixed on the basis of the stability of numerical results with respect to further addition of modes in the spectral representation. For the representation in the $x$-direction it is possible to take whatever number of functions because of their decoupling typical of the linear regime. As known (see GXW), linear solutions having a modal structure of the form:

$$
\begin{aligned}
& \Psi(x, z, t)=\sum_{m} e^{\sigma_{m} t}\left(\sum_{n} \Psi_{m n} g_{n}(z)\right) f_{m}(x), \\
& v(x, z, t)=\sum_{m} e^{\sigma_{m} t}\left(\sum_{n} V_{m n} q_{n}(z)\right) f_{m}(x), \\
& b(x, z, t)=\sum_{m} e^{\sigma_{m} t}\left(\sum_{n} B_{m n} q_{n}(z)\right) f_{m}(x),
\end{aligned}
$$

are characterised by a growth rate distributed in parameter space as shown in Fig. 1. There is a critical value of the Richardson number $\left(\mathrm{Ri}_{c}=0.58\right.$ with the adopted values of parameters) above which the system is stable. Unstable linear circulations are characterised by a sequence of identical clockwise and anticlockwise slanted cells (see an example in Fig. 2). 


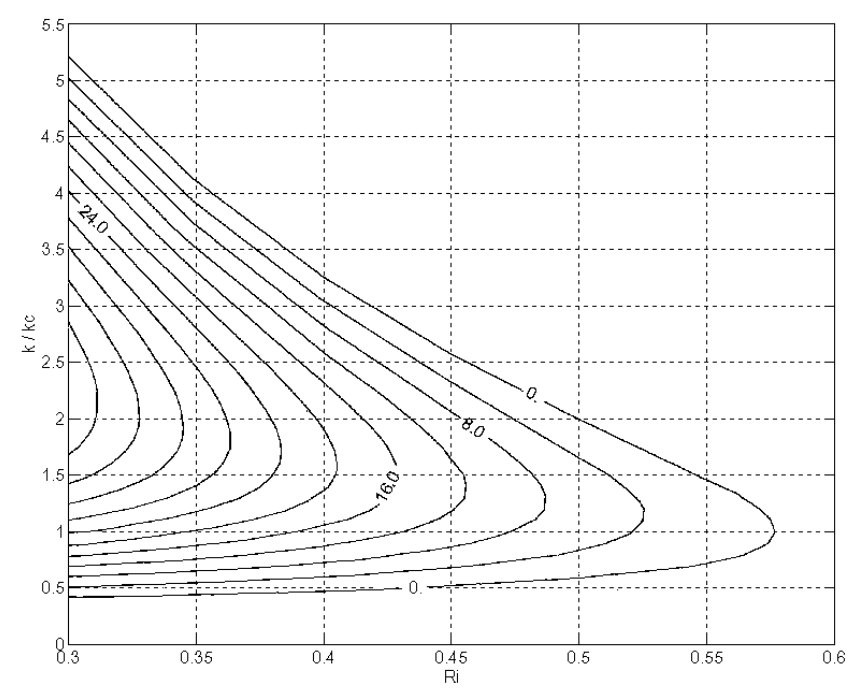

Fig. 1. Spectral model in linear, periodic configuration: growth rate (in $10^{-6} \mathrm{~s}^{-1}$ ) as a function of $k / k$ and $\mathrm{Ri} ; k$ is the wavenumber of the modal solution and $k$ is the critical wavenumber corresponding to the critical Richardson number, $\operatorname{Ri}_{c}\left(k_{c}=6.4 \times 10^{-5} \mathrm{~m}^{-1}\right.$, $\left.\mathrm{Ri}_{C}=0.58\right)$.

\subsection{Nonlinear system}

Integration in time of the nonlinear version of the spectral model, with the adopted spectral representation in terms of 8 modes in $z$ and 11 in $x$ (this configuration has been selected because no relevant variations are observed in the structure and in the amplitude of the solutions adding higher harmonics), leads to nonlinear stationary solutions that are stable with respect to two-dimensional perturbations. Numerical time-integrations initialised in different ways (starting with only the first $x$-harmonic, from the output of a linear run, from random perturbations of the stationary state, etc.) lead to the corresponding nonlinear stationary solutions in a wide range of values of Richardson number. These results confirm previous guesses (see GXW) about the stability of nonlinear stationary solutions found by direct solution of the equations for stationary flow. ${ }^{1}$

In the nonlinear regime higher energies are found in correspondence with wavenumbers that are smaller than those characterised by the highest growth-rates in the lin-

\footnotetext{
${ }^{1}$ Time-integrations show, however, some discrepancies with respect to GXW results. In fact, nonlinear perturbations have nonzero amplitude (measured by means of the area-averaged kinetic energy, $\left.\left(u^{2}+v^{2}+w^{2}\right) / 2\right)$ in a region of parameter space wider than that of linear instability in the range of small wavenumbers. It is difficult to explore this new region of parameter space since, for small wavenumbers, many more $x$-harmonics are needed in order to achieve a correct representation of the solutions. Another difference found is that the amplitude values are exactly two orders of magnitude smaller than the values found by GXW. This order of magnitude corresponds to perturbations of the same "amplitude" as the basic state (in GXW the perturbations have intensities higher than that associated to the basic state).
}

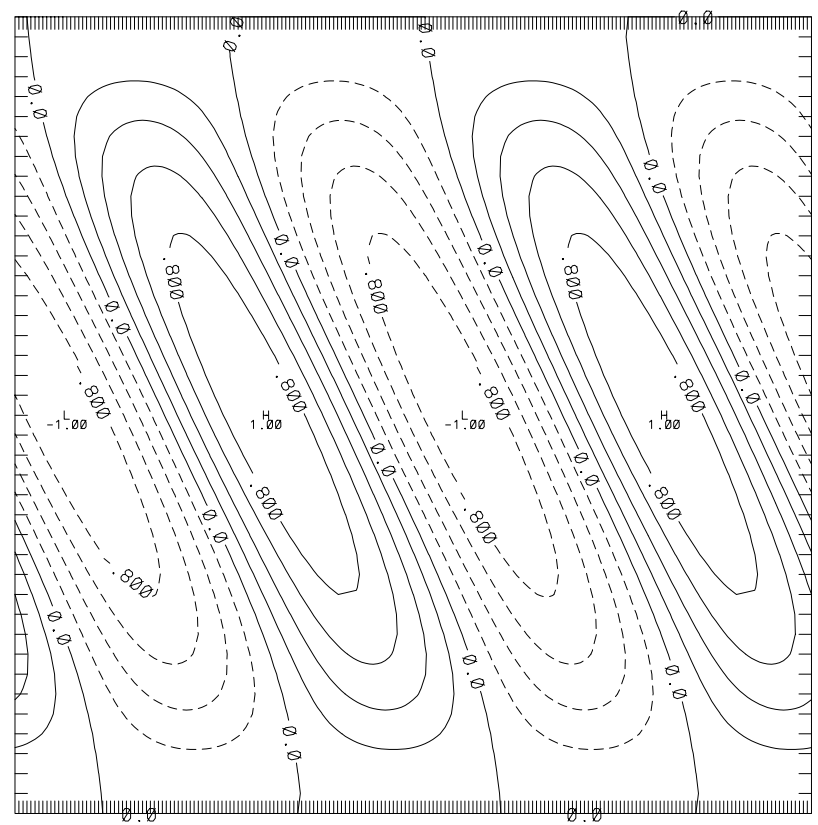

Fig. 2. Spectral model in linear, periodic configuration: vertical cross section of the streamfunction field in the $x-z$ plane; continuous lines represent positive values and dashed lines represent negative values. Clockwise and anticlockwise circulations develop respectively around minima and maxima. Contour interval is 0.2 in arbitrary units. Parameter values are: $\mathrm{Ri}=0.35, k=k_{c}$. The vertical range is $H$ and the horizontal domain covers the largest wavelength of expansion (11a). Since the largest growth rate is that associated to the second $x$-harmonic, $2 k_{c}$ (Fig. 1), two complete waves are present in the fixed domain.

ear regime. Thorpe and Rotunno (1989) put forward the hypothesis that nonlinear equilibration is characterised by a sort of inverse cascading of energy and the progressive emergence of larger scale circulations. Time-integration with the spectral model makes it possible to follow directly the timeevolution of the energy associated to each $x$-harmonic: evidence is that there is no inverse cascading of energy from smaller to higher scales. In fact each $x$-harmonic grows almost independently from the others (see an example in Fig. 3a, where the harmonics are initialised with the same amplitude) and in direct interaction with the basic state until it stabilises by nonlinear self-interaction. As it can be seen in Fig. $3 b$ (where the initial values are derived from the output of a linear run) at the beginning of the integration all the energy in concentrated on the second $x$-harmonic which has the highest linear growth- rate, but, as the run progresses, the first $x$-harmonic, although linearly growing slower than the second $x$-harmonic, reaches a larger amplitude until, finally, the largest energy is concentrated on it. On the other hand, that nonlinear self-interaction of the unstable modes must be the dominant process at least near the threshold of instability, can be understood by qualitative consideration of the perturbative, weakly nonlinear problem. In the near critical regime, in fact, the unstable perturbation coincides with the modifi- 
(a)

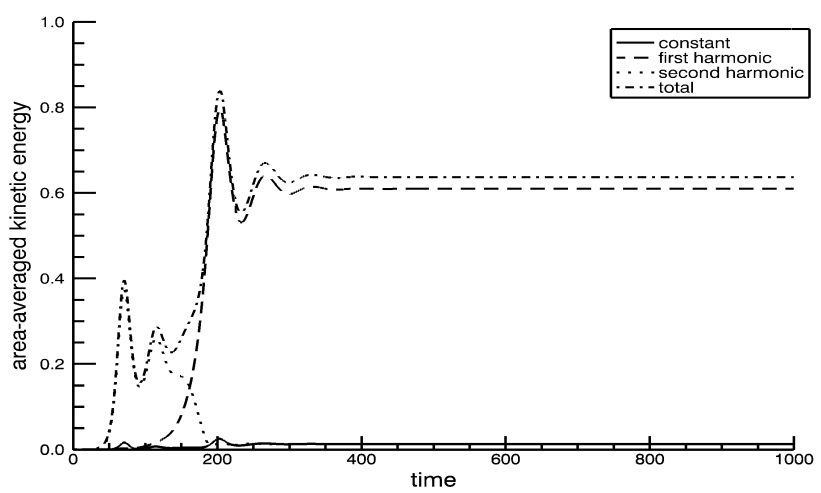

(b)

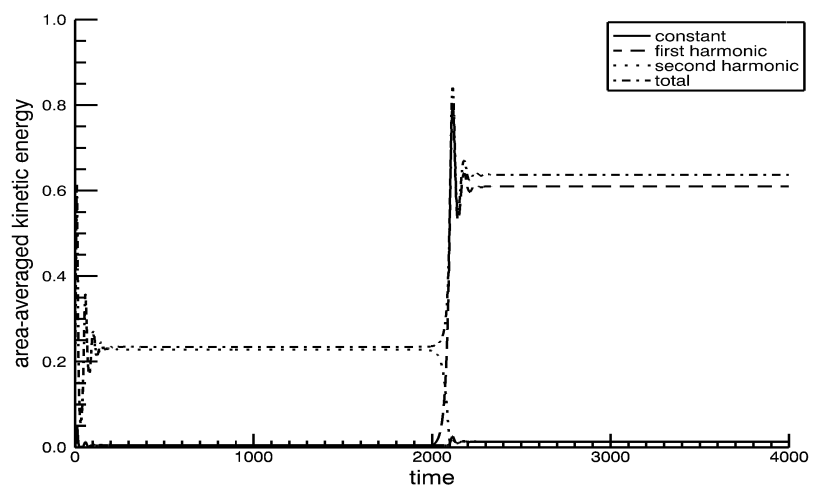

Fig. 3. Spectral model in periodic configuration: nonlinear time evolution of the area-averaged kinetic energy (total and for the first three $x$-harmonics: constant, $k, 2 k$ ): (a) numerical experiment uniformly initialised over all the spectrum; (b) numerical experiment initialised with the output of the linear experiment. Parameter values are: $\mathrm{Ri}=0.35, k=k_{c}$. Energy is measured in $\mathrm{m}^{2} \mathrm{~s}-2$ and time is measured in $3 \times 10^{3} \mathrm{~s}$.

cation of the basic state and the nonlinear correction is the quadratic self- interaction term leading to the typical "Landau stabilisation" (Landau and Lifshitz, 1956) displayed by our solutions.

The structure of nonlinear solutions (see an example in Fig. 4), in the region of parameter-space where it could be tested, is invariably characterised by the "selection" of anticlockwise circulations. This behaviour seems to be in contrast with the previously proposed idea (see Miller, 1984) that the dominance of one kind of circulation on the other may be determined by Prandtl number being different from one. In fact, in the present case (as, for example, in Thorpe and Rotunno, 1989 and Jones and Thorpe, 1992, where the momentum diffusion coefficient is equal to the heat diffusion coefficient) the predominant selection of the anticlockwise circulations is evident. We did not test the structural stability of this result since it was outside the scope of our work.

The difference between clockwise and anticlockwise cells in the nonlinear solution is determined by the presence in the solution of a wind, uniform along the $x$-direction, towards negative $x$-values in the upper part of the integration domain

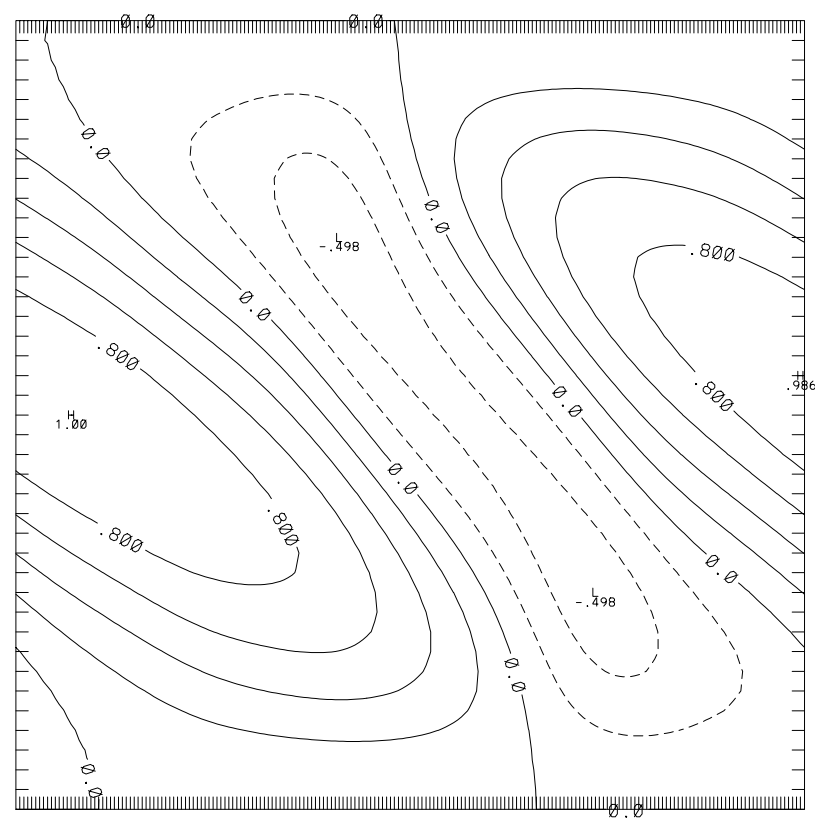

Fig. 4. As in Fig. 2, but for the nonlinear simulation: the solution is normalised at its maximum, that is $1.8 \times 10^{3} \mathrm{~m}^{2} \mathrm{~s}^{-1}$ (contour inter$\mathrm{val}=0.2$ ). The highest energy is associated to the first $x$-harmonic, $k_{c}$, and one only wave is present in the fixed domain.

and positive in its lower part. Since this kind of uniform wind is inhibited by lateral confinement of the flow between two vertical walls, we found interesting to verify the effect of such walls on the stability of the flow. Nonlinear runs were performed with the spectral model adapted to a "box configuration", that is confining the flow both vertically and horizontally by means of walls over which non-slip, conductive conditions are imposed. This was simply done by using in the $x$-expansion the same functions as in the $z$-expansion. We faced, though, problems of numerical stability that lead us to the conclusion that the adopted spectral representation is inadequate (probably because the functions used for this representation are only a complete base and not the natural eigenfunctions of the differential problem in question). As a consequence, in the next Section the box configuration is discussed by means of a finite difference numerical model.

\section{The box configuration}

\subsection{Periodic configuration with finite difference model}

The finite difference model was first developed in the periodic configuration to validate its performance by comparison with the spectral model. By means of the selected staggered grid (shown in Fig. 5) it is possible to introduce the boundary conditions in a straightforward way and the streamfunction derivatives can be defined, by means of centred differences, at the same grid points where $v$ and $b$ are nonzero. In the finite difference formulation the system $(6 a-c)$ is a set of prognostic equations for the vorticity, the velocity in 


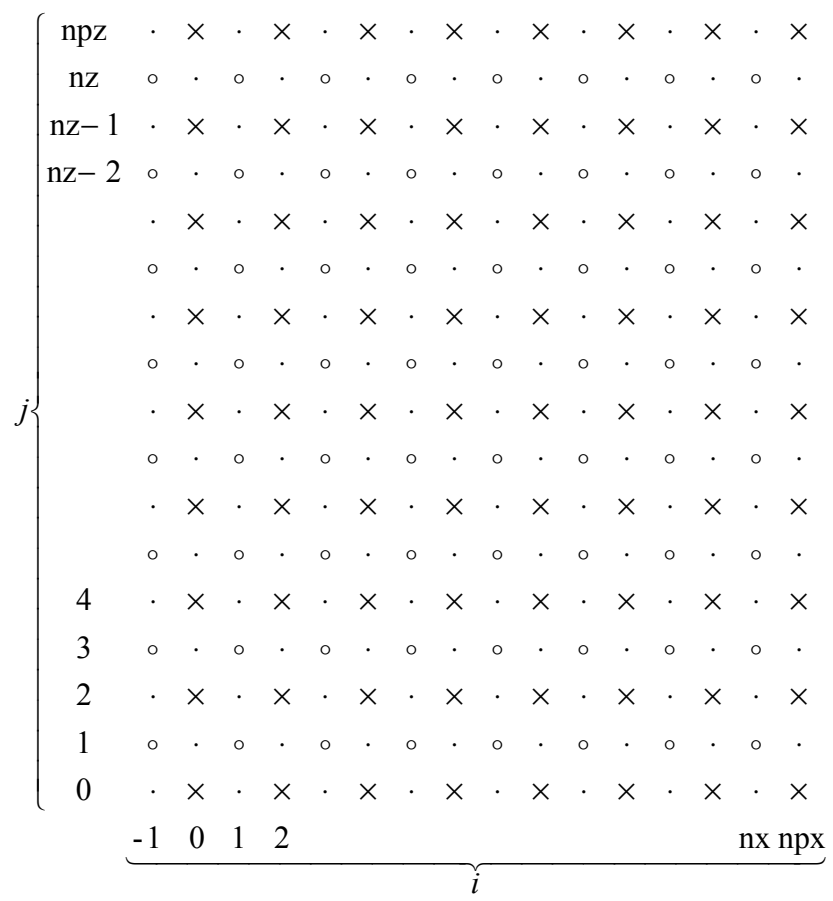

Fig. 5. Finite difference model in periodic configuration: scheme of the staggered grid, where $\Psi$ is nonzero in correspondence of the crosses, and $v$ and $b$ are nonzero in correspondence of the circles; over the dots there is no field. The grid size is $\mathrm{nx}=129, \mathrm{nz}=129$.

the $y$-direction and the buoyancy. The boundary conditions are known only for $\Psi, \partial_{z} \Psi, v$ and $b$ : the known boundary conditions are imposed on the initial streamfunction field; the initial vorticity is then derived by finite-differencing of streamfunction and with (6a) its time-evolution is computed; the updated streamfunction is, finally, obtained from the vorticity with a relaxation method. This procedure is applied at each time-step and the time-integration, for all the prognostic variables, is performed by means of an Euler scheme. Test comparison with the spectral model gives satisfactory results.

\subsection{Box configuration}

As already stated, flow in the box configuration is studied by substituting the previous periodic conditions with non-slip, conductive conditions at vertical walls orthogonal to the $x$ direction (7c). The finite difference scheme adopted in the box configuration is identical to the one of the periodic case, the only differences being that in the new grid the column corresponding to $i=1$ (see Fig. 5) is absent and the finite difference version of conditions (7a), (7c) is imposed in place of the periodicity condition.

Time-integrations, starting from random initial perturbations superimposed on the zonal flow, show that in the linear regime unstable solutions have normal mode structure characterised by a growth rate (Table 1) of the same magnitude and distribution in the parameter space as previously found in the periodic configuration. Here also, nonlinear stationary solutions are stable and their amplitude (measured by means (a)

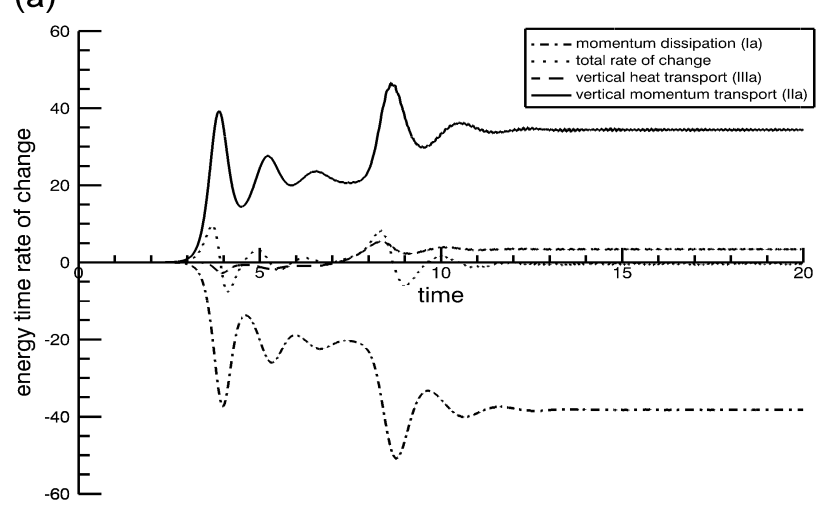

(b)

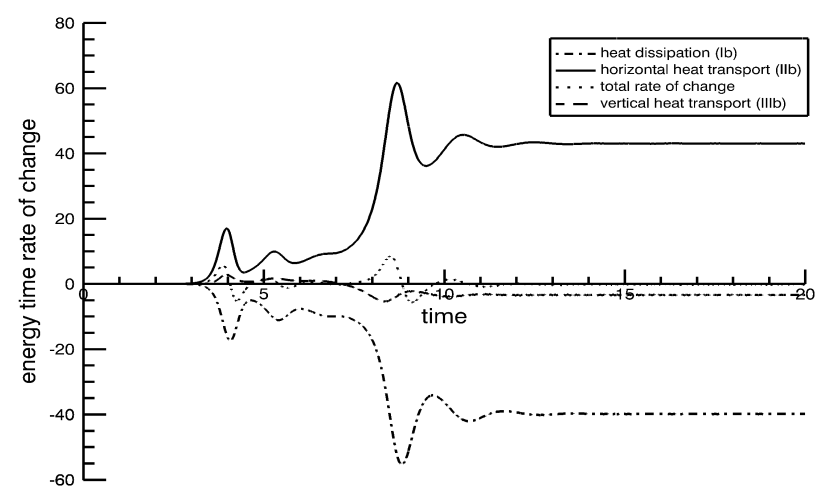

Fig. 6. Finite difference model in periodic, nonlinear configuration: contributions to the time rate of change (in $10^{-6} \mathrm{~m}^{2} \mathrm{~S}^{-3}$ ) of the area-averaged perturbation (a) kinetic and (b) potential energy as a function of time for the simulation with $\mathrm{Ri}=0.35, L=2 \mu / k_{c}$ ( $L$ here represents the length over which the periodic condition is imposed), $\mathrm{E} k=0.01$. The time is measured in $10^{5} \mathrm{~s}$.

of the area-averaged kinetic energy; Table 2) is nonzero in the same region of parameter space where the linear solutions are unstable. An important difference with respect to the periodic case is that in some cases clockwise circulations are dominant, as it can be seen in Table 3 where the symmetry of the solutions is shown as a function of the Richardson number and the width of the horizontal domain.

In the finite amplitude regime, space-scales larger then those characterising the linear regime are observed (as in the periodic configuration).

The structure of the final stationary flow configuration can be quite varied (clockwise or anticlockwise dominant circulation, different number of cells, etc.).

\subsection{The threshold of transition to chaotic behaviour}

In order to identify the range of Richardson number values in which stable stationary solutions dominate the dynamical behaviour of the system, we pushed the Richardson number to values at which the transition to a chaotic regime takes place. In the periodic configuration, the transition to chaotic behaviour was located, within the limits of our numerical ap- 
Table 1. Finite difference model in linear box configuration: growth rate (in $10^{-6} \mathrm{~s}^{-1}$ ) as a function of $L$ and Ri. For a comparison with Fig. 1, $L$ is represented by means of $k / k_{c}$ (where $k_{c}$ is the critical wavenumber of the periodic configuration)

\begin{tabular}{lcccccc}
\hline \multicolumn{7}{c}{$\mathrm{Ri}$} \\
\hline$k / k_{c}$ & 0.35 & 0.4 & 0.45 & 0.5 & 0.55 & 0.6 \\
\hline 4 & $\mathrm{~S}$ & $\mathrm{~S}$ & $\mathrm{~S}$ & $\mathrm{~S}$ & $\mathrm{~S}$ & $\mathrm{~S}$ \\
3.5 & 2 & $\mathrm{~S}$ & $\mathrm{~S}$ & $\mathrm{~S}$ & $\mathrm{~S}$ & $\mathrm{~S}$ \\
3 & 6 & $\mathrm{~S}$ & $\mathrm{~S}$ & $\mathrm{~S}$ & $\mathrm{~S}$ & $\mathrm{~S}$ \\
2.5 & 11 & 1 & $\mathrm{~S}$ & $\mathrm{~S}$ & $\mathrm{~S}$ & $\mathrm{~S}$ \\
2 & 20 & 8 & $\mathrm{~S}$ & $\mathrm{~S}$ & $\mathrm{~S}$ & $\mathrm{~S}$ \\
1 & 26 & 16 & 8 & 2 & $\mathrm{~S}$ & $\mathrm{~S}$ \\
$1 / 2$ & 27 & 17 & 10 & 5 & 0.7 & $\mathrm{~S}$ \\
$1 / 4$ & 24 & 17 & 10 & 5 & 1 & $\mathrm{~S}$ \\
$1 / 8$ & 17 & 12 & 7 & 3 & $\mathrm{~S}$ & $\mathrm{~S}$ \\
$1 / 16$ & 8 & 5 & 2 & $\mathrm{~S}$ & $\mathrm{~S}$ & $\mathrm{~S}$ \\
$1 / 24$ & 3 & 1 & $\mathrm{~S}$ & $\mathrm{~S}$ & $\mathrm{~S}$ & $\mathrm{~S}$ \\
$1 / 36$ & $\mathrm{~S}$ & $\mathrm{~S}$ & $\mathrm{~S}$ & $\mathrm{~S}$ & $\mathrm{~S}$ & $\mathrm{~S}$ \\
\hline
\end{tabular}

$\mathrm{S}=$ stable modal solution

Table 2. As in Table 1, but for the amplitude of the nonlinear solutions measured by means of the area-averaged kinetic energy (in $\mathrm{m}^{2} \mathrm{~s}^{-2}$ )

\begin{tabular}{lcccccc}
\hline & \multicolumn{7}{c}{$\mathrm{Ri}$} \\
\hline$k / k_{c}$ & 0.35 & 0.4 & 0.45 & 0.5 & 0.55 & 0.6 \\
\hline 4 & 0 & 0 & 0 & 0 & 0 & 0 \\
3.5 & 0.01 & 0 & 0 & 0 & 0 & 0 \\
3 & 0.08 & 0 & 0 & 0 & 0 & 0 \\
2.5 & 0.25 & 0.03 & 0 & 0 & 0 & 0 \\
2 & 0.15 & 0.08 & 0 & 0 & 0 & 0 \\
1 & 0.38 & 0.25 & 0.14 & 0.03 & 0 & 0 \\
$1 / 2$ & 0.48 & 0.35 & 0.22 & 0.09 & 0.01 & 0 \\
$1 / 4$ & 0.52 & 0.41 & 0.27 & 0.13 & 0.02 & 0 \\
$1 / 8$ & 0.58 & 0.44 & 0.28 & 0.13 & 0 & 0 \\
$1 / 16$ & 0.68 & 0.45 & 0.21 & 0 & 0 & 0 \\
$1 / 24$ & 0.60 & 0.18 & 0 & 0 & 0 & 0 \\
$1 / 36$ & 0 & 0 & 0 & 0 & 0 & 0 \\
\hline
\end{tabular}

proach, at $\mathrm{Ri} \cong 0.18$ for the spectral model and $\mathrm{Ri} \cong 0.15$ for the finite difference one. In the box configuration (finite difference model only) the transition took place at approximately $\mathrm{Ri} \cong 0.14$. We did not investigate further the chaotic regime since our interest was in stationary states. On the other hand, the values of parameters at which transition occurs are by far non- realistic for atmospheric applications, that are our main geophysical objective.
Table 3. As in Table 1, but for the structure of the nonlinear solutions; the number after the letter refers to the number of waves present in the stationary solution

\begin{tabular}{lccccc}
\hline \multicolumn{5}{c}{$\mathrm{Ri}$} \\
\hline$k / k_{c}$ & 0.35 & 0.4 & 0.45 & 0.5 & 0.55 \\
\hline 3.5 & $\mathrm{C}-1$ & $\mathrm{~N}$ & $\mathrm{~N}$ & $\mathrm{~N}$ & $\mathrm{~N}$ \\
3 & $\mathrm{C}-1$ & $\mathrm{~N}$ & $\mathrm{~N}$ & $\mathrm{~N}$ & $\mathrm{~N}$ \\
2.5 & $\mathrm{C}-1$ & $\mathrm{C}-1$ & $\mathrm{~N}$ & $\mathrm{~N}$ & $\mathrm{~N}$ \\
2 & $\mathrm{~A}-1$ & $\mathrm{~A}-1$ & $\mathrm{~N}$ & $\mathrm{~N}$ & $\mathrm{~N}$ \\
1 & $\mathrm{~A}-1$ & $\mathrm{~A}-1$ & $\mathrm{~A}-1$ & $\mathrm{~A}-1$ & $\mathrm{~N}$ \\
$1 / 2$ & $\mathrm{~A}-2$ & $\mathrm{~A}-2$ & $\mathrm{~A}-2$ & $\mathrm{~A}-2$ & $\mathrm{~A}-2$ \\
$1 / 4$ & $\mathrm{~A}-4$ & $\mathrm{~A}-4$ & $\mathrm{~A}-4$ & $\mathrm{~A}-4$ & $\mathrm{~A}-4$ \\
$1 / 8$ & $\mathrm{~A}-7$ & $\mathrm{~A}-7$ & $\mathrm{~A}-7$ & $\mathrm{~A}-7$ & $\mathrm{~N}$ \\
$1 / 16$ & $\mathrm{~A}-12$ & $\mathrm{~A}-12$ & $\mathrm{~A}-12$ & $\mathrm{~N}$ & $\mathrm{~N}$ \\
$1 / 24$ & $\mathrm{~A}-16$ & $\mathrm{~A}-17$ & $\mathrm{~N}$ & $\mathrm{~N}$ & $\mathrm{~N}$ \\
\hline
\end{tabular}

$\mathrm{C}=$ clockwise dominant circulation

$\mathrm{A}=$ anticlockwise dominant circulation.

$\mathrm{N}=$ zero amplitude circulation.

Table 4. Spectral model in nonlinear, periodic configuration: amplitude of the solution (measured by means of area-averaged kinetic energy in $\mathrm{m}^{2} \mathrm{~s}^{-2}$ ) as a function of and $\mathrm{E} k$

\begin{tabular}{lcccccc}
\hline \multicolumn{7}{c}{$\mathrm{Ri}$} \\
\hline $\mathrm{Ri}$ & 0.0001 & 0.0005 & 0.001 & 0.002 & 0.003 & 0.004 \\
\hline 1 & 0 & 0 & 0 & 0 & 0 & 0 \\
0.9 & $\mathrm{CH}$ & 0.04 & 0.01 & 0 & 0 & 0 \\
0.8 & $\mathrm{CH}$ & $\mathrm{CH}$ & 0.15 & 0.11 & 0.05 & 0.01 \\
0.7 & $\mathrm{CH}$ & $\mathrm{CH}$ & $\mathrm{CH}$ & 0.36 & 0.27 & 0.17 \\
0.6 & $\mathrm{CH}$ & $\mathrm{CH}$ & $\mathrm{CH}$ & $\mathrm{CH}$ & 0.51 & 0.42 \\
0.5 & $\mathrm{CH}$ & $\mathrm{CH}$ & $\mathrm{CH}$ & $\mathrm{CH}$ & $\mathrm{CH}$ & 0.65 \\
0.4 & $\mathrm{CH}$ & $\mathrm{CH}$ & $\mathrm{CH}$ & $\mathrm{CH}$ & $\mathrm{CH}$ & $\mathrm{CH}$ \\
\hline
\end{tabular}

$\mathrm{CH}=$ chaotic regime .

\section{Energy conversions}

\subsection{Definition of energy conversions}

Total perturbation energy, $E$, is defined as a combination of perturbation kinetic energy, $K=\left(u^{2}+v^{2}+w^{2}\right) / 2$, and perturbation potential energy, $P=b^{2} /\left(2 N^{2}\right)$. The rate of change of the area-averaged kinetic, potential, and total perturbation energy is:

$$
\begin{aligned}
& \partial_{t}<K>=\underbrace{\eta<\boldsymbol{v} \cdot \Delta \boldsymbol{v}>}_{I a} \underbrace{-V_{z}<w v>}_{I I a} \underbrace{+<w b>}_{I I I a}, \\
& \partial_{1}<P>=\underbrace{\frac{\eta}{N^{2}}<b \Delta b>}_{I b} \underbrace{-\frac{S^{2}}{N^{2}}<b u>\underbrace{-<b w>}_{I I I b},}_{I I b},
\end{aligned}
$$




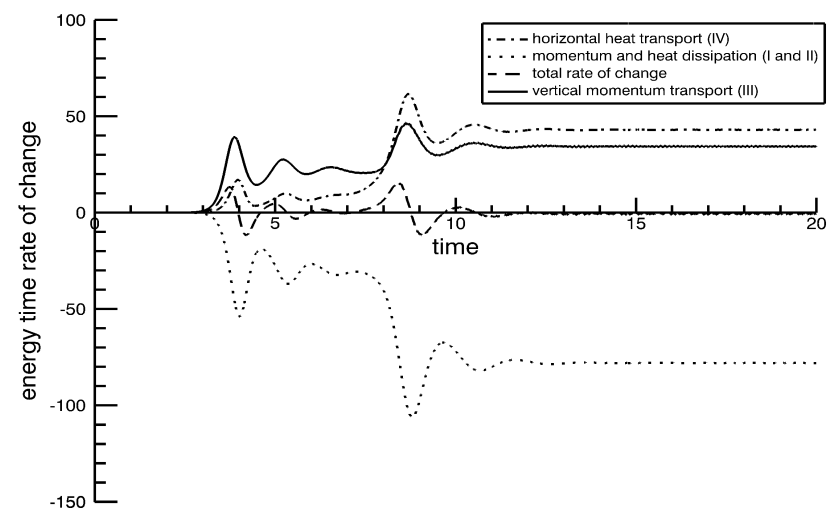

Fig. 7. As in Fig. 6, but for the time rate of change of the areaaveraged total perturbation energy.

$$
\begin{aligned}
\partial_{t}< & E=\underbrace{\eta<\boldsymbol{v} \cdot \Delta \boldsymbol{v}>}_{I} \\
& \underbrace{+\frac{\eta}{N^{2}}<b \cdot \Delta b>\underbrace{-V_{z}<v w>}_{I I I}}_{I I} \underbrace{-\frac{S^{2}}{N^{2}}<b u>}_{I V},
\end{aligned}
$$

where $\boldsymbol{v}=(u, v, w)$ and $<\cdot>$ represents averaging over the entire $x-z$ cross section. Equations $(12 \mathrm{a}-\mathrm{c})$ hold for both periodic and wall-conditions in the $x$-direction. Each equation contains a dissipative contribution (Ia, Ib, I and II). Equations (12a) and (12b) show that there is an exchange between kinetic and potential perturbation energy described by the baroclinic term IIIa (or IIIb) while conversion between basic flow and perturbation kinetic energy is described by the shear term IIa. From Eq. (12c) it is evident that timevariations of total energy are caused by momentum and heat dissipation (respectively I and II), vertical momentum transport (III), and horizontal heat transport (IV).

\subsection{Energy-conversions in linear unstable growth}

Figure 6 shows the time-evolution of the different components of the energy budget: the baroclinic term $<w b>$ removes potential energy (Fig. 6b) and creates kinetic energy (Fig. 6a). However, the principal source of kinetic energy is the shear term $-V_{z}<v w>$ and, consequently, the direct conversion of kinetic energy into kinetic energy. In classical quasi-geostrophic baroclinic instability perturbation energy can be extracted only from the basic state thermal wind and not from the associated vertical shear. Here, however, due to the strongly non-geostrophic nature of the symmetric instability, direct conversion from shear is possible.

\subsection{Energy-conversions in finite amplitude modes}

By means of Eq. (12c) the physical nature of the finite amplitude dynamics can be better understood. In the periodic case (see Fig. 7), it is evident that the limitation to the energy of unstable modes is entirely due to dissipation, while vertical momentum transport and horizontal heat transport cause

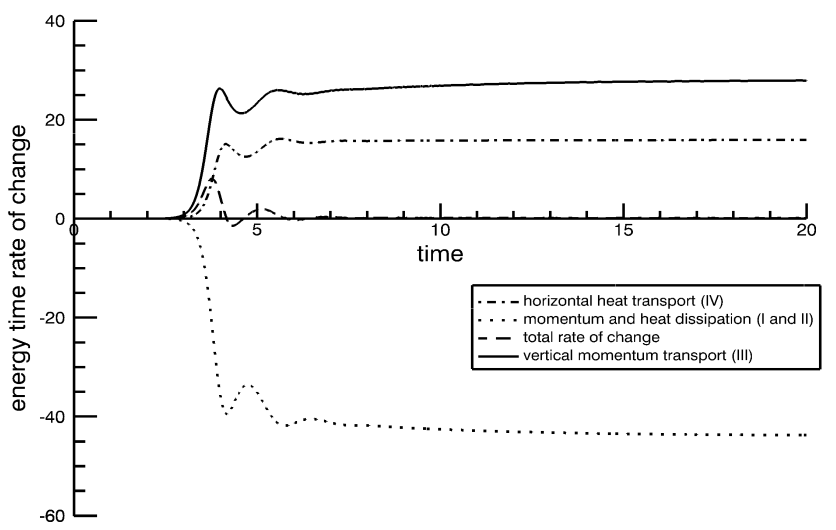

Fig. 8. As in Fig. 7, but with the finite difference model in nonlinear box configuration for the simulation with $\mathrm{Ri}=0.35, L=2 \mu / k(L$ here represents the distance between the vertical walls confining the flow horizontally), $\mathrm{E} k=0.01$.

the growth. As expected (see Sect. 2), this behaviour is very different from linear baroclinic adjustment which would require (see, for example, Emanuel, 1988) the "global" (i.e. at the boundaries of the flow domain) alignment of iso- $V$ surfaces with iso- $M$ ones ( $V$ is the total potential temperature field and $M$ is the total absolute momentum field). In fact, unstable conditions correspond to iso- $M$ surfaces less sloped than iso- $V$ surfaces or, equivalently, to negative potential vorticity $q$ (here defined as $q=J(V, M)$ ). However, with no-slip boundary conditions the global, overall distribution of potential vorticity cannot be changed and remains that of the prescribed basic flow, corresponding to the fixed Richardson number. In the finite amplitude stationary states, $q$ is characterised by structures of the same space scale as the perturbation, in correspondence of which also positive values are reached. The mean value of the field, however, remains the same of the initial condition (uniformly distributed and negative).

From Fig. 7 it is also possible to see that, being vertical velocity typically much smaller than the horizontal one, horizontal heat transport contributes to the energy growth more than vertical momentum transport.

Stabilisation in the box configuration (see Fig. 8) is very similar to that described above for the periodic case. The stationary, final $q$-field is characterised by structures of the same space scale as the perturbation, but the mean value remains the initial one. The presence of walls inhibits the motion in the $x$-direction and this reflects into the energy conversions in which stabilisation is operated by dissipation, but the growth is primarily operated by vertical momentum transport.

In view of the central role played by dissipation in limiting the growth of unstable modes, it is interesting to study regimes of very small viscosity. To this purpose, we searched for the smallest Ekman number associated with stationary solutions by means of the spectral model (in the periodic configuration) where the fundamental $x$-harmonic is equal to the critical one. Table 4 shows, in the parameter-space of the 


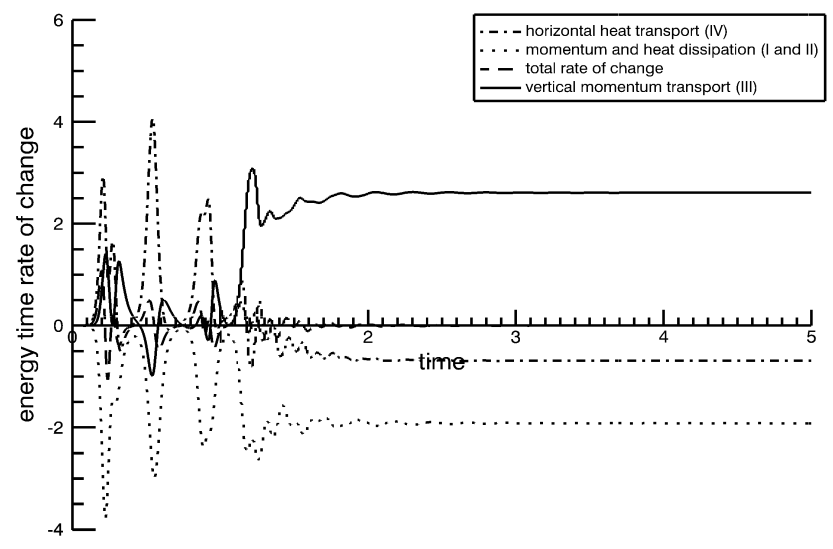

Fig. 9. As in Fig. 7, but with the spectral model in nonlinear, periodic configuration for the simulation with $\mathrm{Ri}=0.9, k=k_{c}, \mathrm{E} k=$ 0.0005 . The time is now measured in $3 \times 10^{6} \mathrm{~s}$

system, the area-averaged kinetic energy of the perturbation stationary solution when a stable state is reached and, otherwise, the indication of a chaotic regime. As it can be seen, when the Ekman number is reduced also the interval between zero amplitude and chaotic regime decreases. The smallest Ekman number to which we could associate a stationary solution is 0.0005 , with a Richardson number equal to 0.9 . Time-evolution of energy (Fig. 9) reveals that not only dissipation operates to contrast the growth of perturbations, but also the horizontal heat transport gives a contribution. In all the analysed cases, there is no correlation between the sign of the stabilised $q$-field and the particular circulation developed in the same region.

\section{Conclusions}

In this paper we analyse linear and nonlinear symmetric flows of a non-hydrostatic stratified rotating fluid, forced by a constant thermal wind and dissipated by Laplacian friction. The study is performed by means of numerical timeintegration and analysis of energy conversions in time. With the type of forcing adopted, in the unstable regime, the flow does not support any kind of linear baroclinic adjustment (drift of the basic state towards conditions of neutrality with respect to linear instability). Consequently, in terms of energy conversions it can achieve nonlinear equilibration only by balancing the energy injection due to the instability of the basic zonal wind with dissipation, while the nonlinear selfinteraction of the growing modes neutralises the baroclinic instability.

Our analysis confirms that in a vast range of values of Richardson number (between the threshold of linear instability of the zonal flow and that of transition to chaotic behaviour) stable nonlinear stationary solutions exist that are reached by stabilisation of linearly unstable modes.

The space-scale typical of such finite amplitude solutions is larger then the one characterising fastest growing linear modes. Analysis of the mechanism of scale-selection in the periodic case shows unstable modes that are slower growing and of larger space-scale than the most unstable ones, eventually stabilise to a larger amplitude. It is the efficiency of nonlinear self-interaction of growing modes, rather than nonlinear mode-mode coupling, to select the scale of the mode eventually dominating the flow.

The physical mechanism of stabilisation is characterised by the fact that, under conditions of linear instability, the final stable state shows a potential vorticity field with structures of the same space-scale as the perturbation circulations, while the mean potential vorticity in the flow domain remains unchanged (as dictated by the boundary conditions). In fact, energy analysis shows that the global energy-stabilisation is entirely operated by dissipation. The mechanism seems to be robust as it operates efficiently with both periodic and box boundary conditions, in a vast range of flow parameters.

The main features of the modelled symmetric circulations are in general agreement with what we observe in precipitation bands, except for the time-scale typical of the transition (from linear growth to nonlinear stabilisation) which is rather long with respect to what we observe in the atmosphere. Other mechanisms (for example, direct transfer of vorticity from the basic flow into the symmetric circulation) may be competitive. Also the application of the above described models to parameterisation of symmetric flow in synoptic scale atmospheric models seems problematic, since no variational criterion could be identified. In order to achieve practically useful results, further studies seem necessary as, in particular:

- Generalization to boundary conditions allowing for global adjustment, i.e. feed-back onto the zonal flow.

- Generalization to three-dimensional turbulent flow in which the background shear can operate more directly and dissipation is more realistically modelled. Both generalisations are under study.

Acknowledgements. This work was supported by Italian Space Agency under grant N. ARS-99-60, and by Bologna University for partial fulfilment of "Dottorato di Ricerca" by R. Mantovani. We thank the group of Dynamical Meteorology of ISAO-CNR in Bologna for technical support and useful discussions.

\section{References}

Aebischer, U. and Schär, C.: Low-level potential vorticity and cyclogenesis to the lee of the Alps, J. Atmos. Sci., 55, 186-207, 1998.

Emanuel, K. A.: Inertial instability and mesoscale convective systems. Part I: Linear theory of inertial instability in rotating viscous fluids, J. Atmos. Sci., 36, 2425-2449, 1979.

Emanuel, K. A.: Observational evidence of slantwise convective adjustment, Mon. Wea. Rev., 116, 1805-1816, 1988.

$\mathrm{Gu}, \mathrm{W} ., \mathrm{Xu}, \mathrm{Q}$., and Wu, R.: Three-dimensional instability of nonlinear viscous symmetric circulations, J. Atmos. Sci., 55, 31483158, 1998. 
Jones, S. C. and Thorpe, A. J.: The three-dimensional nature of "symmetric" instability, Quart. J. Roy. Meteor. Soc., 118, 227258, 1992.

Landau, L. D. and Lifshitz, E. M.: Fluid Mechanics, AddisonWesley, New York, 1956.

Miller, T. L.: The structures and energetics of fully nonlinear symmetric baroclinic waves, J. Fluid Mech., 142, 343-362, 1984.

Schultz, D. M. and Schumacher, P. N.: The use and misuse of conditional symmetric instability, Mon. Wea. Rev., 127, 2709-2732,
1999.

Stone, P. H.: Baroclinic adjustment, J. Atmos. Sci., 35, 561-571, 1978.

Thorpe, A. J. and Rotunno, R.: Nonlinear aspects of symmetric instability, J. Atmos. Sci., 46, 1285-1299, 1989.

Tudur, E. and Ramis, C.: The environments of significant convective events in the western Mediterranean, Wea. Forecasting, 12, 294-306, 1997. 